\title{
Hypothesis Article: Free SARS-CoV-2 spike protein S1 particles may act as a factor of COVID-19 pathogenesis
}

\author{
Andrey Letarov ${ }^{1}$, Vladislav Babenko ${ }^{2}$, and Eugene Kulikov ${ }^{3}$ \\ ${ }^{1}$ Federal Research Center "Fundamentals of Biotechnology", Russian Academy of Sciences \\ ${ }^{2}$ FSBI Federal Research and Clinical Center of Physical-Chemical Medicine of the Federal \\ Medical Biological Agency of Russia \\ ${ }^{3}$ Winogradsky Institute of Microbiology, FRC "Fundamentals of Biotechnology", Russian \\ Academy of Sciences
}

July 16, 2020

\begin{abstract}
The disbalance of the renin-angiotensin system was suggested to play an important role in the pathogenesis of the COVID-19 disease. Previously it has been shown that ACE2 expression in downregulated in the murine model in response to SARS-CoV infection and may be also induced by the recombinant spike protein alone. We hypothesize that the soluble SARS-CoV-2 spike protein S1 subunits shed from the infected cells and from the virions in vivo may bind to the ACE2 receptor and trigger ACE2 downregulation. Decreased ACE2 activity on the background of the constant or increased ACE activity in the lungs may lead to the prevalence of angiotensin II effects over angiotensin(1-7) connected to increased thrombosis, inflammation and pulmonary damage.
\end{abstract}

Keywords: SARS-CoV-2, renin-angiotensin system, Spike protein S1 subunit, COVID-19 pathogenesis.

The pandemic of COVID-19 disease that caused already hundreds of thousands of fatalities worldwide is caused by the coronavirus SARS-CoV-2 that is most probably transited from an animal host to humans in late 2019 (Chen, Liu \& Guo, 2020). The virus is highly related to the bat Coronavirus RaTG13, however, the existence of a transient host (probably a pangolin) was suggested (Zhang, Wu \& Zhang, 2020). The adaptation of SARS-CoV-2 virus to human host is believed to be mostly due to acquisition of the novel sequence of the tail spike (S) protein receptor binding domain (RBD) that efficiently recognizes human angiotensin converting enzyme 2 (ACE2). Although a related SARS-CoV coronavirus also recognizes ACE2 as its cellular receptor (Chen, Liu \& Guo, 2020), only 8 out of 14 critical a.a. residues involved into the interaction of the ACE2 and RBD are conserved between SARS-CoV and SARS-CoV-2 viruses (Zhang, Wu \& Zhang, 2020).

The coronavirus entry into the host cell requires cleavage of the $\mathrm{S}$ protein by the host proteases at the junction between S1 and S2 subunits (Chen, Liu \& Guo, 2020). This processing can take place after the virion attachment by the cell surface protease TMPRSS2 or in the lysosomal compartment after the internalization of the virus (Chen, Liu \& Guo, 2020; Shang et al., 2020). The proteolytic processing of $\mathrm{S}$ protein allows S1 subunit to dissociate to trigger the S2 subunit rearrangement to extended post-fusion conformation required for initiation of the fusion of the viral and lysosomal membranes (Walls et al., 2017).

In some coronaviruses, however, the $\mathrm{S}$ protein processing may take place during the virion assembly in the Golgi compartment. Such processing requires furin protease recognition site to be present at the S1 and S2 subunits junction (Shang et al., 2020). In SARS-CoV-2 the insertion of 4 a.a. sequence PRRA after the a.a. 675 in $\mathrm{S}$ protein that lead to the formation of the furin cleavage site RRAR (Zhang, Wu 
\& Zhang, 2020). This leads to efficient processing of the S protein in the virus-producing cell (Hoffmann, Kleine-Weber \& Pöhlmann, 2020; Shang et al., 2020). The pre-processing of the spike protein before the virus release makes the infection more efficient (Shang et al., 2020) and potentially may allow part of the virus particles to penetrate into the host cells directly at the plasma membrane without entering to the lysosomal compartment (Freeman, Peek, Becker, Smith \& Denison, 2014). More efficient host cell entry taken together with higher affinity of its RBD (compared to RBD of SARS-CoV that lacks efficient furin processing site) may compensate SARS-CoV-2 for decreased availability of the RBDs for ACE2-receptor binding (Shang et al., 2020). Indeed, on most of the SARS-CoV-2 S protein trimers two out of three RBDs remain in closed conformation in which they are shielded from both host immunity factors (e. g. antibodies) and from receptor recognition, while in SARS-CoV virus all three RBDs are in "open" conformation on most of the spikes (Wrapp et al., 2020).

As in any virus pneumonia, the pathology of COVID-19, as well of SARS, is believed to be mainly due to the virus killing of the lung epithelial cells, and, even to larger extent, due to immune-mediated mechanisms (Chen, Liu \& Guo, 2020; Matricardi, Dal Negro \& Nisini, 2020).

However, the recognition of ACE2 as the receptor by both SARS-CoV and SARS-CoV-2 raised a hypothesis that in addition to the aforementioned mechanisms (that may be further enhanced by a secondary bacterial infection) the pathology of COVID-19 and of SARS may in large extent rely on the virus-induced disbalance of the renin-angiotensin system (RAS)(Gheblawi et al., 2020; Verdecchia, Cavallini, Spanevello \& Angeli, 2020).

ACE2 is a cell-surface metalloprotease (carboxypeptidase) that converts angiotensin I decapeptide into angiotensin(1-9) nonapeptide, in contrast to ACE enzyme, that produced out of angiotensin I the physiologically active angiotensin II nonapeptide. Angiotensin(1-9) can be converted by ACE enzyme into angiotensin(1-7) heptapetide. Angiotensin II also may be processed into angiotensin(1-7) by ACE2(Gheblawi et al., 2020; Verdecchia, Cavallini, Spanevello \& Angeli, 2020). The physiological activity of angiotensin II leads to vasoconstriction and increased arterial pressure but it also promotes inflammation reactions, blood coagulation and thrombosis, fibrosis capillary permeability and edema. Angiotensin ${ }_{1-7}$ has basically opposite effect decreasing inflammation, thrombosis, fibrosis and causes vasodilatation (Gheblawi et al., 2020; Verdecchia, Cavallini, Spanevello \& Angeli, 2020). Therefore, upregulation of ACE and/or downregulation of ACE2 lead to increased pulmonary damage.

It has been demonstrated that SARS-CoV infection decreases ACE2 expression in murine model (Gheblawi et al., 2020; Kuba et al., 2005; Verdecchia, Cavallini, Spanevello \& Angeli, 2020) suggested that virus attachment to ACE2 molecules lead to their removal from the cell surface via co-endocytosis with the virus thus diminishing the ACE2 activity. This leads to the imbalance between angiotensin II and angiotensin(1-7) in the lung tissue and to increases thrombosis and pulmonary damage (Gheblawi et al., 2020; Verdecchia, Cavallini, Spanevello \& Angeli, 2020).

From our point of view, the direct mechanical removal of the ACE2 molecules by the virion attachment is unlikely to contribute significantly to the overall activity of the enzyme in the lungs. Such an effect would require simultaneous virus attachment to large fraction of the ACE2 producing cells (such as alveocytes II). We believe that at that high virus load the direct damage of the epithelial cells would not be compatible with the patient survival, though the reported mortality even in severe COVID-19 cases is moderate (Chen, Liu \& Guo, 2020; Matricardi, Dal Negro \& Nisini, 2020).

Interestingly, in (Kuba et al., 2005) study the reduction of ACE2 in mice could be induced not only by SARS-CoV infection but also by the recombinant SARS-CoV spike protein. The mice pre-treated (i.p.) with this spike protein did not show significant pathology but if these animals were acid instilled, the spike protein pre-treatment lead to increased severity of the lung damage (Kuba et al., 2005).

Therefore, the spike protein if present in significant molar excess in respect to the viral particles may in fact mediate the down regulation of ACE2 and RAS imbalance. It has been shown that S proteins of murine coronaviruses and of SARS-CoV are indeed delivered onto the cell surface, presumably, as a side product 
of the virus assembly and release process. These molecules may induce some physiological effect such as micropinocytosis and/or membrane fusion of the neighbor cells (Freeman, Peek, Becker, Smith \& Denison, 2014), however S protein been a membrane protein, remains attached to the infected cell membrane. It worth to mention that $\mathrm{S}$ protein is also present on the viral particles released from the infected cells into the medium.

It has been recently demonstrated by direct cryo-electron and negative-stain electron microscopy (Liu et al., 2020 ) that on the in vitro cultured SARS-CoV-2 particles retaining their infectivity most of S protein trimers are in the post-fusion conformation. In the other words, the S1 subunits dissociation took place before the actual receptor binding (apparently the biological activity of these virions was assured by the remaining fraction of S trimers that did not yet shot in vain). Although in the conditions of the study, the S1 subunits dissociation could be triggered by the conditions during the gradient centrifugation used to purify the virus (Liu et al., 2020). Nevertheless, this result demonstrates that in SARS-CoV-2 processed spikes S1 subunit dissociation can be triggered by relatively mild conditions that are compatible with the virus viability, or occur spontaneously. We hypothesize that substantial amount of free soluble S1 subunits may be shed from the infected cells and virions (Fig 1).

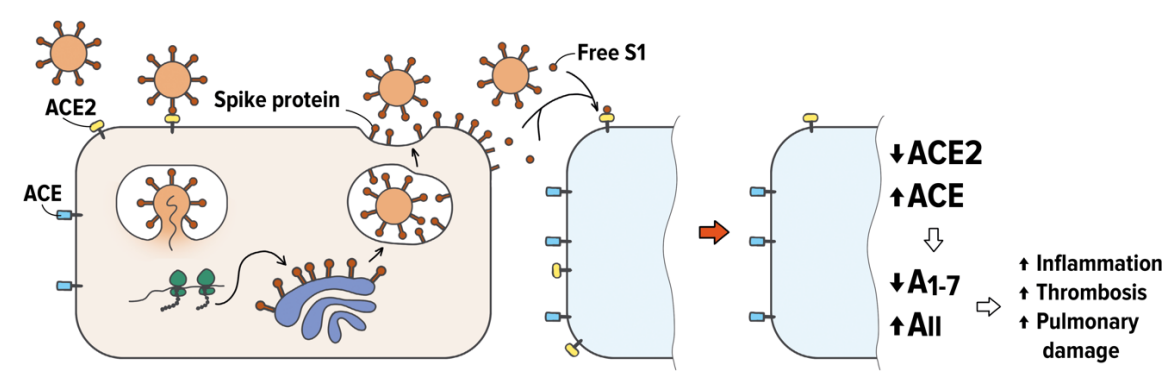

\section{Figure 1.}

Hypothesis of involvement of free S1 subunits of SARS-CoV-2 spike protein into COVID-19 induced

Binding to the receptor via their intact RBDs these molecules may induce ACE2 downregulation and the downstream deleterious effects as it was suggested by (Gheblawi et al., 2020; Verdecchia, Cavallini, Spanevello \& Angeli, 2020).

If our hypothesis turns true, the release of free S1 particles from the infected cells and virions should reduce the virus infectivity towards the cells neighboring to the infected cells. Therefore, two stages of the SARSCoV2 infection in the lungs can be predicted:

1. The virus infects certain loci where the production of free S1 protein causes the downregulation of ACE2 in the non-infected cells in the proximity of the viruse-infected cells. Simultaneously the expression of ACE2 on more distant cells, that did not yet encountered S1 particles, can be increases due to the effect of interferon produced in the infected cells that has been shown to stimulate ACE2 expression (Ziegler et al., 2020). The local disbalance of the angiotensin II/ angiotensisn (1-7) levels also may elicit compensatory ACE2 increase in the cells not yet affected by the virus (though such compensatory circuit is not yet described, it would be logical to suggest it may exist). Therefore, the virus spread for longer distances (e.g. to another alveoles) would be facilitated, however the virus induced tissue damage would be limited.

2. When larger amount of the tissue is infected, the greater quantities of free S1 is produced, and the RAS disbalance on the organ or organism level is induced. This causes the deleterious effects such as increased inflammation, thrombosis and pulmonary damage as it was suggested previously (Gheblawi et al., 2020; Verdecchia, Cavallini, Spanevello \& Angeli, 2020). Simultaneously the virus production should be decreased due to downregulation of ACE2 production in the infected areas of the lungs. 
This model corresponds well to the known clinical traits of COVID-19 (Siordia, 2020; Zhao et al., 2020): involvement of large areas of the lungs with frequent bi-lateral pneumonia (stages I of our model), reported relatively good patients state despite significant part of the lungs involved according to radiological examination data that is followed by abrupt deterioration of the patients conditions in next hours or days (late stage I and stage II).

The proposed model suggests that the clinical interventions suggested to maintain RAS balance (Sriram \& Insel, 2020) should have synergistic effect with protease inhibitors, specially with furin inhibitors. Although the in vitro data suggest that lack of the furin pre-processing of SARS-CoV-2 S protein can be compensated by the post-attachment processing by other proteases (Shang et al., 2020), our model suggests that furin inhibition will not only decrease the virus infectivity but will also decrease the shed of the free S1 particles from both virions and infected cells and blunt the infection effect over RAS.

It also should be mentioned that free S1 molecules may represent a target for COVID-19 therapy or prevention. After the separation of S1 subunit from S2 stem the potential epitopes free of the glycans shielding the external surface of the complete $\mathrm{S}$ protein ${ }^{13}$ become exposed. So the immunization by the recombinant proteins that would elicit the antibodies response against these conserved and unprotected epitopes may lead to the sequestration of free S1 molecules to immune complexes and their elimination thus reducing the probability of severe COVID-19 pneumonia.

\section{Acknowledgements}

Authors a grateful to Ms. Ksenya Sayfulina from Moscow State University of Psychology and Education for the help with the figure creation.

\section{Conflict of interest}

Authors declare no conflict of interest.

\section{References}

Chen Y, Liu Q, \& Guo D (2020). Emerging coronaviruses: Genome structure, replication, and pathogenesis. J Med Virol 92:418-423.

Freeman MC, Peek CT, Becker MM, Smith EC, \& Denison MR (2014). Coronaviruses induce entryindependent, continuous macropinocytosis. MBio 5.

Gheblawi M, Wang K, Viveiros A, Nguyen Q, Zhong JC, Turner AJ, et al. (2020). Angiotensin-Converting Enzyme 2: SARS-CoV-2 Receptor and Regulator of the Renin-Angiotensin System: Celebrating the 20th Anniversary of the Discovery of ACE2. Circ Res 126: 1456-1474.

Hoffmann M, Kleine-Weber H, \& Pöhlmann S (2020). A multibasic cleavage site in the spike protein of SARS-CoV-2 is essential for infection of human lung cells. Molecular Cell.

Kuba K, Imai Y, Rao S, Gao H, Guo F, Guan B, et al. (2005). A crucial role of angiotensin converting enzyme 2 (ACE2) in SARS coronavirus-induced lung injury. Nat Med 11: 875-879.

Liu C, Yang Y, Gao Y, Shen C, Ju B, Liu C, et al. (2020). Viral architecture of SARS-CoV-2 with post-fusion spike revealed by Cryo-EM. Biorxiv.

Matricardi PM, Dal Negro RW, \& Nisini R (2020). The first, holistic immunological model of COVID-19: Implications for prevention, diagnosis, and public health measures. Pediatr Allergy Immunol.

Shang J, Wan Y, Luo C, Ye G, Geng Q, Auerbach A, et al. (2020). Cell entry mechanisms of SARS-CoV-2. Proc Natl Acad Sci U S A 117: 11727-11734.

Siordia JA, Jr. (2020). Epidemiology and clinical features of COVID-19: A review of current literature. J Clin Virol 127: 104357. 
Sriram K, \& Insel PA (2020). A hypothesis for pathobiology and treatment of COVID-19: The centrality of ACE1/ACE2 imbalance. Br J Pharmacol.

Verdecchia P, Cavallini C, Spanevello A, \& Angeli F (2020). The pivotal link between ACE2 deficiency and SARS-CoV-2 infection. European Journal of Internal Medicine.

Walls AC, Tortorici MA, Snijder J, Xiong X, Bosch BJ, Rey FA, et al. (2017). Tectonic conformational changes of a coronavirus spike glycoprotein promote membrane fusion. Proc Natl Acad Sci U S A 114: $11157-11162$.

Wrapp D, Wang N, Corbett KS, Goldsmith JA, Hsieh CL, Abiona O, et al. (2020). Cryo-EM Structure of the 2019-nCoV Spike in the Prefusion Conformation. bioRxiv.

Zhang T, Wu Q, \& Zhang Z (2020). Probable Pangolin Origin of SARS-CoV-2 Associated with the COVID-19 Outbreak. Curr Biol 30: 1578.

Zhao D, Yao F, Wang L, Zheng L, Gao Y, Ye J, et al. (2020). A comparative study on the clinical features of COVID-19 pneumonia to other pneumonias. Clin Infect Dis.

Ziegler CGK, Allon SJ, Nyquist SK, Mbano IM, Miao VN, Tzouanas CN, et al. (2020). SARS-CoV-2 Receptor ACE2 Is an Interferon-Stimulated Gene in Human Airway Epithelial Cells and Is Detected in Specific Cell Subsets across Tissues. Cell 181: 1016-1035 e1019. 